\title{
1 Characterization of BRS1 functions in plant stress responses
}

3 State Key Laboratory of Crop Stress Biology for Arid Areas, College of Agronomy,

4 Northwest A\&F University, Yangling, Shaanxi 712100, China

5

* Correspondence: Shengbao Xu, xushb@nwsuaf.edu.cn

6

7

8

9

10

11

12

13

14

15

16

17

18

19

20

21

22

23 
Background: Brassinosteroid-insensitive 1 suppressor 1 (BRS1), is a serine carboxypeptidase that mediates brassinosteroid signaling and participates in multiple developmental processes in Arabidopsis. However, little is known about the precise role of BRS1 in this context.

29 Results: In this study, we analyzed transcriptional and proteomic profiles of

30 Arabidopsis seedlings overexpressing BRS1 and found that this gene is involved in both

31 biotic and abiotic stress responses and redox regulation. Further proteomic evidence

32 shows that BRS1 regulates cell redox by indirectly interacting with cytosolic NADP+-

33 dependent isocitrate dehydrogenase (cICDH). We identified two novel splice products

34 of $B R S 1$, which might play important roles in development and stress responses in 35 plants.

36 Conclusions: This study highlights the role of BRS1 in plant redox regulation and 37 stress responses, which extends our understanding of extracellular serine 38 carboxypeptidases.

39 Keywords: Serine carboxypeptidase, Stress response, Redox, Arabidopsis 


\section{Background}

Serine carboxypeptidases (SCP) are a class of eukaryotic proteolytic enzymes, belonging to the $\alpha / \beta$ hydrolase family. These enzymes contain the highly conserved catalytic amino acid triad, Ser-Asp-His [1]. In Arabidopsis, 54 SCP-like genes have been identified and categorized into three classes [2, 3]. The functions of the majority of these genes have not been described.

BRS1 belongs to class II of the Arabidopsis SCP family [1]. Its overexpression can rescue the receptor mutant of brassinosteroid (BR), bril-5, indicating BRS1 might play an important role in BR signaling [4, 5]. BRS1 has five close homologs, of which three can suppress bril-5 developmental defects [6]. In contrast, no significant phenotypes have been identified in single or double mutants of either BRS1 or its homologs $[4,6$, 7]. This suggests that BRS1 and its homologs are functionally redundant in Arabidopsis, making difficulties to clarify the precise roles of BRS1.

$$
\text { Increasing evidence has revealed that SCP and SCP-like (SCPL) proteins play crucial }
$$
roles in the regulation of stress responses, including regulating wound healing [8], programmed cell death and response to pathogen infections $[9,10]$. BRS1 contains a signal peptide and localizes to the extracellular space $[4,5]$. Since the extracellular space is central in regulating plant stress responses [11-13], it is likely BRS1 might mediate some of these signaling pathways.

Environmental stress leads to the accumulation of reactive oxygen species (ROS) in plant cells and results in oxidative stress [14-16]. Therefore, to adapt to environmental stress, it is vital to control redox homeostasis to maintain normal cellular metabolism, and/or trigger programmed cell death $[17,18]$.

In this study, transcriptomic and proteomic analyses demonstrate a role for BRS1 in 
71 biotic and abiotic stress responses. Our results indicate BRS1 participates in redox regulation via interaction with cICDH, shedding new light onto the function of SCPs in

73 plant stress responses.

74 Results

75 Seedling developmental phenotypes of $B R S 1$

76 BRS1-1D was over-expressed BRS1 with an enhancer in BRS1 promoter [4, 5], 77 showing larger rosette leave (Fig. 1a) and a longer hypocotyl (Fig. 1b, c, d and e).

78 However, we saw no visible phenotypes with the BRS1 knockdown mutant, brs1-1 (Fig.

79 1), consistent to no significant phenotype was observed in BRS1 mutant [4-7].

\section{Analysis of the transcriptomes of BRS1 seedlings}

We performed RNA sequencing on the BRS1 seedlings (Fig. 1b). A total of 21,873 transcripts were identified, of which 180 differentially expressed genes (DEGs, fold change $\geq 2.0$, FDR-adjusted P-value $<0.05$, FPKM $\geq 1$ ) were identified in the brs $1-1 D$ seedlings when compared to wild type. This included increased expression of 114 genes and decreased expression of 66 genes (Supplementary Table S1). In contrast, no gene significantly changed its transcription in mutant brs $1-1$ seedlings, except BRS1 itself (Supplementary Table S1). This is consistent with the finding of no visible morphological difference shown in brs1-1 plants (Fig. 1).

However, this was an unusual result as we expected some genes in the network would be affected in the brs 1-1 plants. We, therefore, used our RNA sequencing data to investigate the transcription of mutated BRS1 in our seedlings. The transcription of BRS1 in the mutant brs1-1 was decreased significantly due to the insertion of a thymidine at position 786 in the first intron (Fig. 2a and b), which resulted in a missplicing event (Fig. 2c). In contrast, the transcriptional level of BRS1 increased by 
around 20 times in the brs $1-1 D$ mutant (Fig. 2a), which is consistent with the presence of four copies of CaMV 35S enhancers inserted in the promoter region of BRS1 (Fig. 2b) [4].

Interestingly, the transcriptional level of the first exon in brs 1-1 maintained a similar level to that of the wild type (Fig. 2c), indicating there is a not feedback loop to enhance BRS1 transcription. We also saw no increased expression of the homologs of BRS1 when BRS1 expression was disrupted (Supplementary Figure S1).

Notably, we detected novel splice variants in $\operatorname{brs} 1-1 D$, which retained multiple introns compared to the wild type (Fig. 2c). The two new splice products (4 and 5) made up a lower proportion of the total $B R S 1$ transcripts in brs $1-1 D$, then the level still equal to the total transcripts of BRS1 in wild type (Fig. 2c), indicating these novel splice products may have important functions.

\section{Overexpression of BRS1 alters multiple stress responses}

Gene Ontology (GO) enrichment analysis of DEGs from brs 1-1D found a significant enrichment in genes associated with responding to salicylic acid (SA) and jasmonic acid (JA) (Fig. 3). We also found enrichment in genes associated with both biotic (innate immune responses, bacterium, fungus, and chitin) and abiotic (water deprivation, cold and hyperosmotic salinity) stresses (Fig. 3). In agreement with these findings, genes associated with redox regulation and cell death were also enriched. These results strongly suggest that BRS1 participates in the responses to both abiotic and biotic stressors.

\section{BRS1 regulates redox-related proteins}

We next performed proteomic analysis on seedlings using two-dimensional difference gel electrophoresis (2D-DIGE) (Fig. 4a). 19 proteins were assigned as differentially 
expressed proteins, and 14 proteins were identified, of which 5 proteins were involved in redox regulation (Table 1), supporting that BRS1 is involved in redox regulation.

121 The 2D-DIGE revealed two spots (6 and 7), which were most changed compared to

122 wild type, showing a decrease and increase in $\operatorname{brs} 1-1 D$, respectively (Fig. 4). These

123 spots corresponded to the same protein, the cytosolic NADP+-dependent isocitrate

124 dehydrogenase (cICDH, at 1 g65930) (Table 1), a critical redox regulator [19]. Together,

125 these data suggest that BRS1 is involved in redox regulation

\section{BRS1 participates in redox regulation by interacting with cICDH}

127 To confirm that BRS1 regulates cICDH, the enzyme activity of cICDH was measured

128 in BRS1 mutants. We found that cICDH activity was significantly increased in brs 1-1D

129 compared to wild type plants (Fig. 5a), whilst no change was seen in brs 1-1 plants.

130 These findings are consistent with our earlier results that showed only overexpression

131 of BRS1 altered transcription. Altogether this suggests that BRS1 regulates the activity 132 of cICDH.

133 To understand how the secretory protein BRS1 could regulate cICDH, which is

134 localized to the cytosol, the cellular localization of BRS1 was evaluated. We observed 135 expression of BRS1 at the membrane and cell wall of mature epidermal cells (Fig. 5b),

136 consistent with localization to the extracellular space $[4,5]$. Unexpectedly, we also

137 found cICDH-GFP to be localized to the membrane of Nicotiana benthamiana leaves

138 (Fig. 5c), indicating a potential interaction might occur in this area. However, yeast 139 two-hybrid and pull-down assays found no direct interaction between cICDH and BRS1

140 (Supplementary Table S2), indicating that BRS1 regulates cICDH indirectly.

\section{Discussion}

142 BRS1, as a member of SCP, its role is relative clear in SCP family, and its function in 
143 plant growth and development has been demonstrated previously [4, 6, 7]. In this study,

144 the transcriptome suggested its important role in stress response, highlighting BRS1 as

145 the apoplastic protease, share the important function in biotic and abiotic stress defense $146[13,20]$.

147 Our results suggest that the BRS1-related stress response is involved in SA and JA 148 signaling (Fig. 3). These are key hormones required for the induction of plant defenses 149 in response to pathogens and insects [21, 22]. Similarly, an apoplastic SCPL in rice, 150 OsBISCPL1, also induces a stress response via SA and JA signaling [9], indicating 151 SCPLs may use a common mechanism to regulate stress responses.

152 The trigger effector production by apoplastic proteases used be the key mechanism 153 to induce plant stress response [13]. Our results showed that systemic stress response 154 was induced upon BRS1 over-expression (Fig. 3). Thus this pathway is likely involved 155 in plant defense more generally, rather than being limited to a specific pathogen.

156 Consistent with this notion, we found BRS1 regulates redox homeostasis, and 157 therefore plays a critical role in controlling apoplastic ROS [13]. ROS regulation is vital 158 to induce widespread stress responses, and essential for SA and JA signaling in response 159 to various stressors $[23,24]$

160 The ICDHs catalyze the production of NADPH, which is important for redox 161 regulated cell metabolism and promoting redox signaling in response to oxidative stress $162[25,26] . \mathrm{cICDH}$ is responsible for more than $90 \%$ of total $\operatorname{ICDH}$ activity $[25,27]$. 163 Therefore, this enzyme plays a crucial role in maintaining redox homeostasis in the cell, 164 and consequently, defense responses [19]. Exactly how BRS1 participates in this 165 pathway still needs further investigation.

166 The knockdown mutant of BRS1 does not have any significant phenotypes $[4,6,7]$; 
consistently, we found no transcriptional changes in the mutant brs 1-1 (Supplementary

168 Table S1). However, this finding suggests that there are no pathways dependent on

169 BRS1. In contrast, we observed many phenotypes and identified numerous alterations

170 in gene expression upon overexpression of BRS1 [7]. The existence of multiple

171 redundant homologs in Arabidopsis may explain this result [4, 6, 7]. However, in this

172 study the concurrence of the novel splice variants, phenotypes and activity of cICDH

173 in brs 1-1D (Fig. 2c), implying a splicing-based functional variation of BRS1, which

174 may play a special role in those highly redundant gene family to overcome their

175 redundancy nature in changing environments.

\section{Conclusions}

177 In this study, transcriptomic and proteomic analyses revealed that BRS1 plays a role in

178 regulating plant responses to biotic and abiotic stress. We find that BRS1 likely

179 participates in redox regulation in cells through indirect interaction with cICDH.

180 Altogether, our work sheds new light on the roles played by SCPs in biotic and abiotic

181 stress responses.

182 Methods

\section{Plant Materials and Growth Condition}

184 The plant materials used in this study are as follows: Wild-type Wassilewskija (WS2)

185 and mutants of BRS1 (brs 1-1 and brs 1-1D). The brs $1-1 D$ is generated by crossing WS2

186 with an activation-tagging line bri1-5 brs $1-1 D$ (CS6127) from the laboratory of Jia Li

187 (Lanzhou University, Lanzhou, China) [4, 5, 7]. The mutant brs $1-1$ also came from the

188 laboratory of Jia $\mathrm{Li}$, which was originally obtained from the Wisconsin Arabidopsis

189 knockout pool. This mutant identified insertion a thymidine at $786 \mathrm{bp}$ in first intron

190 through our RNA sequencing and has been used in previous research [7]. Theses seeds 
were grown in growth chamber at $22^{\circ} \mathrm{C}$ under $16 \mathrm{~h}$ light $/ 8 \mathrm{~h}$ dark conditions (light intensity $100 \mu \mathrm{mol} \cdot \mathrm{m}^{-2} \cdot \mathrm{s}^{-1}$, humidity $60 \%$ ) for two weeks after germination to perform

193 phenotypic observation. Seedlings of the three materials were cultured on half-strength

194 Murashige and Skoog (1/2 MS) agar medium (supplemented with 1\% (w/v) sucrose

195 and $0.8 \%(\mathrm{w} / \mathrm{v})$ agar, $\mathrm{PH} 5.6-5.8)$ in the same chamber and culture conditions, and total

196 protein and RNA were extracted seven days after germination. T-DNA insertion mutant 197 icdh-2 (SALK_056247) was ordered from the Salk Institute collection of Arabidopsis

198 Biological Resource Center (ABRC) and verified by genotyping as previously 199 described [19].

\section{RNA Extraction and Gene Expression Profiling}

201 Seven-day-old WS2, brs 1-1, and brs 1-1D seedlings grown vertically on a 1/2 MS plate

202 were frozen in liquid nitrogen, and triplicate of each material were collected. Total RNA

203 was extracted from whole seedlings using a Tiangen RNAprep pure Plant Kit, and its 204 quality was evaluated with Thermo Scientific NanoDrop2000. The RNA sequencing 205 was completed by Biomarker company (Beijing, China).

\section{Analysis of Transcriptome Data}

207 Raw transcriptome sequencing datas cleaned using Trimmomatic (v 0.36) under default 208 parameters. The clean reads were aligned to the TAIR 10 reference genome using 209 HISAT2 (v 2.1.0) and the expression of genes were profiled using StringTie (v1.3.3) $210[28,29]$. The reads in the AT4G30610 (BRS1) gene region using StringTie for transcript 211 assembly and reads coverage using "genomeCoverageBed" statistics in BEDtools (v $212 \quad 2.29 .0)$.

213 Identification of Differentially Expressed Genes (DEGs) and GO enrichment 214 analysis 
215 Identification all of differential genes in brs 1-1 and brs 1-1D compared to control WS2,

216 respectively. The corrected read count data of genes were imported into the R package

217 DESeq2 (v1.26.0) to identify DEGs with the standard of a fold change $\geq 2.0$, a false

218 discovery rate (FDR)-adjusted P-value $<0.05$, and expression $(\mathrm{FPKM} \geq 1)$ in at least

219 one sample for each comparison [30].

220 The GO descriptions were obtained by AnnotationHub (“AH75734”), and used the 221 R package clusterProfiler (v3.14.0) with the "enrichGO" function for GO enrichment 222 analysis. The statistical significance of the enrichment of GO was examined using the 223 hypergeometric distribution test, followed by multiple-test correction using the 224 Benjamini-Hochberg method. GO terms with q -value $<0.01$ for further enrichment 225 analysis.

\section{Protein Preparation for Fluorescent Two Dimension Difference Gel}

\section{Electrophoresis (2D-DIGE) Analysis}

228 Seven-day-old seedlings ( $1 \mathrm{~g})$ were harvested and ground to a fine powder in liquid 229 nitrogen and further mixed with $4 \mathrm{~mL}$ ice-cold extract buffer $(20 \mathrm{mM}$ Tris-HCl, PH 8.0, $2301 \mathrm{mM}$ EDTA, $20 \mathrm{mM} \mathrm{NaCl}, 5 \mathrm{mM} \mathrm{MgCl} 2,10 \mathrm{mM}$ DTT, $2 \mathrm{mM}$ phenylmethanesulfonyl 231 fluoride, $1 \mu \mathrm{g} / \mathrm{mL}$ leupeptin, $10 \mu \mathrm{g} / \mathrm{mL}$ aprotinin, $1 \mu \mathrm{g} / \mathrm{mL}$ chymostatin and $1 \%$ 232 phosphorylase inhibitor mixture). The supernatant was collected by centrifugation at $23318,000 \mathrm{~g}$ for $20 \mathrm{~min}$ at $4^{\circ} \mathrm{C}$, and the pellet was resuspended in $3 \mathrm{~mL}$ extract buffer for 234 repeat extraction. The combined supernatant was supplemented with chilled acetone to $23580 \%(\mathrm{~V} / \mathrm{V})\left(4\right.$ times volume acetone of the supernatant) and incubated at $-20^{\circ} \mathrm{C}$ 236 overnight to precipitate proteins. Proteins were pelleted by dissolved in $100 \mu \mathrm{L}$ lysis 237 buffer (7 M urea, $2 \mathrm{M}$ thiourea, 4\% w/v CHAPS, $20 \mathrm{mM}$ Tris- $\mathrm{HCl}, \mathrm{pH} 8.5$ ), and the 238 debris was removed by centrifugation at $18,000 \mathrm{~g}$ for $20 \mathrm{~min}$. Finally, the $\mathrm{pH}$ of protein 
samples was adjusted to 8.5 with $\mathrm{HCl}$ and $\mathrm{NaOH}$, and the concentration of proteins

240 were determined as previously described Bio-Rad Bradford method using BSA as a

241 standard [31]. The final proteins underwent 2D-DIGE immediately or were stored in

242 aliquots at $-80^{\circ} \mathrm{C}$. For each sample, at least quadruplicate protein preparations were 243 performed.

\section{D-DIGE and Image Analysis}

245 According to the manufacturer's instructions (GE Healthcare), the equivalent amounts 246 of brs 1-1 and brs 1-1D proteins were labeled with Cy3 and Cy5 minimum fluorescent 247 dyes (400 pmol dye/50 $\mu \mathrm{g}$ protein), respectively. The internal standard WS2 protein was 248 labeled with CY2 and mixed with two different labeled proteins in equal amounts. 249 Adjust the mixed-labeled protein to a total volume of $450 \mu \mathrm{L}$ with rehydration buffer 250 (8 M urea, $13 \mathrm{mM}$ DTT, 4\% $\mathrm{w} / \mathrm{v}$ CHAPS, 0.5\% Pharmalyte $\mathrm{pH} 3-10$ ), and then load 251 on an IPG test strip holder containing an IPG test strip with $24 \mathrm{~cm} \mathrm{pH} \mathrm{4-7} \mathrm{linear} \mathrm{gradient}$ 252 (GE Healthcare). Experimental methods of isoelectric focusing and SDS-PAGE as 253 previously described [32]. To minimize systemic and inherent biological differences, it 254 is recommended to combine four independent protein preparations for each sample [33].

255 Fluorescent images of gels were scanned by Typhoon 9400 scanner (GE Healthcare) 256 and the images were analyzed using DeCyder 6.5 software in accordance with the 257 DeCyder User Manual (GE Healthcare) [32]. Approximately 2000 spots were detected 258 in each image, and then spots that showed significant differential expression were 259 determined by ANOVA and Student's t-test $(\mathrm{p}<0.05) .19$ spots with significant 260 differential expression were selected for mass spectrometric identification.

\section{Protein Identification}

262 Coomassie brilliant blue staining was performed on the scanned 2-D-DIGE gel, and 
then differential protein spots were found by position comparison, but it was difficult

264 to detect proteins with low background expression. Therefore, a 2-DE gel prepared with

$2651 \mathrm{mg}$ of internal standard protein was used for staining to show spots that could not be 266 determined from the 2D-DIGE gel.

267 After 19 differential protein spots were excised from 2-D-DIGE gel, each spot was 268 destained in destaining buffer ( $25 \mathrm{mM}$ ammonium bicarbonate, $50 \% \mathrm{v} / \mathrm{v}$ acetonitrile).

269 Destained spots were dehydrated by acetonitrile and spun-dry, and digested with 270 sequencing grade modified trypsin (Roche) at $37^{\circ} \mathrm{C}$ for $16 \mathrm{~h}$. The matrix-assisted laser271 desorption ionization (MALDI) mass spectra were produced on an Ultroflex II MALDI 272 time-of-flight/time-of-flight mass spectrometer (MALDI-TOF/TOF MS) (Bruker 273 Daltonics, Germany) with use of FlexAnalysis 2.4 software. After tryptic peptide 274 masses were transferred to a BioTools 3.0 interface (Bruker Daltonics), peptide mass 275 fingerprintings (PMFs) were searched against the NCBInr protein database 276 (http://www.ncbi.nlm.nih.gov/; NCBInr 20071214; 5,742,110 sequences) by use of 277 Mascot software2.2.03(http://www.matrixscience.com; Matrix Science, London,U.K.).

\section{Enzyme activities}

279 Seven-day-old of seedlings $(0.1 \mathrm{~g})$ were ground to a fine powder in liquid nitrogen and 280 mixed with the extract buffer (1 mL 0.1 M NaH2PO4 (pH 8.0), $5 \mathrm{mM} \mathrm{MgCl} 2,14 \mathrm{mM}$ 281 2-mercaptoethanol). Vortex the homogenate, centrifuge at $12,000 \mathrm{~g}$ for 5 minutes to 282 remove insoluble materials, and measure ICDH activity by spectrophotometry [19, 34]. 283 Determination of protein concentration as previously described Bio-Rad Bradford 284 method using BSA as a standard [31].

\section{List of abbreviations}

286 SCP: Serine carboxypeptidase; BRS1: Brassinosteroid-insensitive 1 suppressor 1; ROS: 
Reactive oxygen species; DEGs: Differentially expressed genes; FPKM: Fragments per

288 kilobase of exon per million fragments mapped; SA: Salicylic acid; JA: Jasmonic acid;

289 cICDH: Cytosolic NADP+-dependent isocitrate dehydrogenase

Declarations

291 Ethics approval and consent to participate

292 Not applicable.

293 Consent for publication

294 Not applicable.

295 Availability of data and materials

296 All data generated or analyzed in this study are included in this article and the 297 supplemental files. The raw data of RNA sequencing were submitted to the NCBI 298 database with the bioproject ID: PRJNA657702.

299 Competing interests

300 The authors declare that they have no competing interests.

$301 \quad$ Funding

302 This work was funded by the National Key Research and Development Program of 303 China (2016YFD0101602) and the National Natural Science Foundation of China 304 (31370318). This Funding was not involved in the design of the study and collection, 305 analysis, and interpretation of data and in writing the manuscript.

306 Author Contributions

307 SX conceived and designed the research and contributed to writing and revising the 308 manuscript. DZ performed experimental work and data analysis, and wrote and revised 309 the manuscript. PZ performed bioinformatics analysis. All authors have read and 310 approved the manuscript. 
312 We thank Jia Li (Lanzhou University, Lanzhou, China) for kindly providing the mutants 313 of BRS1.

\section{References}

315 1. Fraser CM, Rider LW, Chapple C. An expression and bioinformatics analysis of 316 the Arabidopsis serine carboxypeptidase-like gene family. Plant Physiol.

2. Tripathi LP, Sowdhamini R. Cross genome comparisons of serine proteases in Arabidopsis and rice. BMC Genom. 2006;7:200-30.

3. Zhu D, Chu W, Wang Y, Yan H, Chen Z, Xiang Y. Genome-wide identification, classification and expression analysis of the serine carboxypeptidase-like protein family in poplar. Physiol Plantarum. 2018;162(3):333-52.

4. Li J, Lease KA, Tax FE, Walker JC. BRS1, a serine carboxypeptidase, regulates BRI1 signaling in Arabidopsis thaliana. Proc Natl Acad Sci U S A. 2001;98(10):5916-21.

5. Zhou A, Li J. Arabidopsis BRS1 is a secreted and active serine carboxypeptidase. J Biol Chem. 2005;280(42):35554-61.

6. Wen J, Li J, Walker JC. Overexpression of a serine carboxypeptidase increases carpel number and seed production in Arabidopsis thaliana. Food Energy Secur. 2012;1(1):61-9.

7. Deng Q, Wang X, Zhang D, Wang X, Feng C, Xu S. BRS1 Function in facilitating lateral root emergence in Arabidopsis. Int $J$ Mol Sci. 2017;18(7):1549.

8. Moura DS, Bergey DR, Ryan CA. Characterization and localization of a woundinducible type I serine-carboxypeptidase from leaves of tomato plants (Lycopersicon esculentum Mill.). Planta. 2001;212(2):222-30.

9. Liu H, Wang X, Zhang H, Yang Y, Ge X, Song F. A rice serine carboxypeptidaselike gene OSBISCPL1 is involved in regulation of defense responses against biotic and oxidative stress. Gene. 2008;420(1):57-65.

10. Domínguez F, González MC, Cejudo FJ. A germination-related gene encoding a serine carboxypeptidase is expressed during the differentiation of the vascular tissue in wheat grains and seedlings. Planta. 2002;215(5):727-34.

11. Zhu JK. Abiotic stress signaling and responses in plants. Cell. 2016;167(2):31324.

12. Zhou JM, Zhang Y. Plant immunity: danger perception and signaling. Cell. 2020;181(5):978-89.

13. Wang Y, Wang Y, Wang Y. Apoplastic proteases: powerful weapons against pathogen infection in plants. Plant Communications. 2020;100085.

14. Apel K, Hirt H. Reactive oxygen species: metabolism, oxidative stress, and signal transduction. Annu Rev Plant Biol. 2004;55:373-99.

15. Waszczak C, Carmody M, Kangasjärvi J. Reactive oxygen species in plant signaling. Annu Rev Plant Biol. 2018;69:209-36.

16. Sies H, Berndt C, Jones DP. Oxidative stress. Annu Rev Biochem. 2017;86(1):715748.

17. Suzuki N, Koussevitzky S, Mittler R, Miller G. ROS and redox signalling in the 
response of plants to abiotic stress. Plant Cell Environ. 2012;35(2):259-70.

18. Qi J, Wang J, Gong Z, Zhou J-M. Apoplastic ROS signaling in plant immunity. Curr Opin Plant Biol. 2017;38:92-100.

19. Mhamdi A, Mauve C, Gouia H, Saindrenan P, Hodges M, Noctor G. Cytosolic NADP-dependent isocitrate dehydrogenase contributes to redox homeostasis and the regulation of pathogen responses in Arabidopsis leaves. Plant Cell Environ. 2010;33(7):1112-23.

20. Stael S, Van Breusegem F, Gevaert K, Nowack MK. Plant proteases and programmed cell death. J Exp Bot. 2019;70(7):1991-5.

21. Browse J. Jasmonate passes muster: a receptor and targets for the defense hormone. Annu Rev Plant Biol. 2009;60:183-205.

22. Zhang Y, Li X. Salicylic acid: biosynthesis, perception, and contributions to plant immunity. Curr Opin Plant Biol. 2019;50:29-36.

23. Noctor G, Reichheld JP, Foyer $\mathrm{CH}$. ROS-related redox regulation and signaling in plants. Semin Cell Dev Biol. 2018;80:3-12.

24. Pieterse CMJ, Van der Does D, Zamioudis C, Leon-Reyes A, Van Wees SCM. Hormonal modulation of plant immunity. Annu Rev Cell Dev Biol. 2012;28(1):489-521.

25. Hodges M, Flesch V, Gálvez S, Bismuth E. Higher plant NADP+-dependent isocitrate dehydrogenases, ammonium assimilation and NADPH production. Plant Physiol Bioch. 2003;41(6):577-85.

26. Marino D, González EM, Frendo P, Puppo A, Arrese-Igor C. NADPH recycling systems in oxidative stressed pea nodules: a key role for the NADP+ -dependent isocitrate dehydrogenase. Planta. 2007;225(2):413-21.

27. Hodges M. Enzyme redundancy and the importance of 2-oxoglutarate in plant ammonium assimilation. J Exp Bot. 2002;53(370):905-16.

28. Kim D, Langmead B, Salzberg SL. HISAT: a fast spliced aligner with low memory requirements. Nat Methods. 2015;12(4):357-60.

29. Pertea M, Pertea GM, Antonescu CM, Chang T-C, Mendell JT, Salzberg SL. StringTie enables improved reconstruction of a transcriptome from RNA-seq reads. Nat Biotechnol. 2015;33(3):290-5.

30. Love MI, Huber W, Anders S. Moderated estimation of fold change and dispersion for RNA-seq data with DESeq2. Genome Biol. 2014;15(12):550-70.

31. Bradford MM. A rapid and sensitive method for the quantitation of microgram quantities of protein utilizing the principle of protein-dye binding. Anal Biochem. 1976;72:248-54.

32. Xu SB, Yu HT, Yan LF, Wang T. Integrated proteomic and cytological study of rice endosperms at the storage phase. J Proteome Res. 2010;9(10):4906-18.

33. Barceló-Batllori S, Kalko SG, Esteban Y, Moreno S, Carmona MC, Gomis R. Integration of DIGE and bioinformatics analyses reveals a role of the antiobesity agent tungstate in redox and energy homeostasis pathways in brown adipose tissue. Mol Cell Proteomics. 2008;7(2):378-93.

34. Galvez S, Bismuth E, Sarda C, Gadal P. Purification and characterization of chloroplastic NADP-isocitrate dehydrogenase from mixotrophic tobacco cells. Plant Physiol. 1994;105(2):593-600. 
a. Comparison of the phenotypes of wild type WS2, BRS1 knockdown mutant brs 1-1 and BRS1 overexpression allele brs 1-1D at 14 days after germination (DAG). Plants were grown in a growth chamber at $22^{\circ} \mathrm{C}$ under long-day conditions. Scale bar $=1 \mathrm{~cm}$.

b. The phenotypes of WS2, brs1-1 and brs1-1D seedlings grown on 1/2 MS medium under long-day conditions. Photos captured 7 DAG. Scale bar $=1 \mathrm{~cm}$.

c. The phenotypes of WS2, brs1-1 and brs 1-1D seedlings grown on 1/2 MS medium in the dark. Photos captured 5 DAG. Scale bar $=1 \mathrm{~cm}$.

d. Comparison of hypocotyl lengths of seedlings grown as pictured in (b). Means \pm

412 standard error (SE) are shown from three independent experiments, $\mathrm{n} \geq 20$ in each 413 experiment.

414 e. Comparison of the hypocotyl lengths of seedlings grown as pictured in (c). Means \pm

415 SE are shown from three independent experiments, $n \geq 20$ in each experiment. Asterisks 416 indicate a statistically significant difference based on the Student's t-test $(* \mathrm{P}<0.05)$.

417 Figure 2. Differences in gene sequences and $B R S 1$ transcription in wild type and BRS1 mutants

a. Transcription levels (FPKM value) of BRS1 in WS2, brs 1-1 and brs 1-1D as measured 420 by RNA sequencing. All results are expressed as mean \pm standard deviation (SD). The 421 asterisks indicate a statistically significant difference (Student's t-test, $* \mathrm{P}<0.05$ ).

b. Summary of the differences in gene sequences between WS2, brs 1-1 and brs1-1D. 423 BRS1 has nine exons and eight introns. A thymidine base (red) is inserted in the first 424 intron of brs 1-1, and the 4 X 35S enhancer is inserted in the promoter of BRS1 in brs 1- 
c. The transcription of BRS1 was measured in WS2, brs1-1and brs1-1D. The counts of different reads of $B R S 1$ and their distribution in genes are shown above. Data is from three biological replicates per sample. The types of different BRS1 transcripts present in the different samples are shown. The percentage value shows the ratio of individual transcripts to total transcripts. The red box highlights the normal transcript of $B R S 1$ in TAIR10.

Figure 3. Enrichment analysis of DEGs in transcripts of brs1-1D compared to wild type Analysis of GO terms associated with DEGs between brs 1-1D and WS2 seedlings (qvalue $<0.01)$. The first red column represents DEGs that are up-regulated in brs $1-1 D$ compared to WS2; the second blue column represents DEGs that are down-regulated. Terms found enriched in the up-regulated DEGs are divided into four categories (red vertical lines). The term enriched in the down-regulated DEGs is indicated with the blue vertical line. Gene ratio indicates the ratio of DEGs clustered into different terms, divided by the total number of DEGs.

Figure 4. Identification of two isoforms of cICDH and comparison of protein expression levels

a. Representative 2D-DIGE image of the two most significant differential protein spots 444 in seedlings from WS2, brs 1-1 and brs 1-1D. Samples from WS2, brs 1-1 and brs 1-1D 445 were labeled with Cy2 (blue), Cy3 (green), and Cy5 (red), respectively. White arrows 446 indicate differential protein spots. brs 1-1D. The height of the pink circled area represents the level of protein expression. 
450 a. Total extractable ICDH activity from wildtype (WS2 and Col-0) seedlings and a 451 range of mutant lines: brs 1-1D, brs 1-1 and icdh-2. Means and SE of eight independent 452 extracts are shown. Asterisks indicate a statistically significant difference based on 453 Student's t-test $(* \mathrm{P}<0.05)$.

454 b. Subcellular localization of BRS1. Root tips from transgenic Col-0 plants expressing 455 35S-BRS1-GFP were used to visualize the subcellular localization of BRS1-GFP by 456 confocal microscopy.

457 c. Subcellular localization of ICDH. Nicotiana benthamiana leaves transiently 458 expressing 35S-ICDH-GFP were used to observe the subcellular localization of ICDH459 GFP by confocal microscopy.

460 Table 1. List of differentially expressed proteins identified by mass spectrometry (MS)

\begin{tabular}{|c|c|c|c|c|}
\hline $\begin{array}{l}\text { No } \\
\text {. }\end{array}$ & $\begin{array}{l}\text { Chromosome } \\
\text { locus }\end{array}$ & Matched protein & Biological process & Expression \\
\hline 1 & AT3G08590 & $\begin{array}{l}\text { 2,3-Biphosphoglycerate-independent } \\
\text { phosphoglycerate mutase } 2\end{array}$ & $\begin{array}{l}\text { Carbohydrate } \\
\text { metabolism }\end{array}$ & 1.18 \\
\hline 2 & AT3G57610 & Adenylosuccinate synthetase & AMP biosynthesis & 0.87 \\
\hline 3 & AT3G54050 & $\begin{array}{l}\text { Chloroplastic fructose } 1,6- \\
\text { bisphosphate phosphatase }\end{array}$ & $\begin{array}{l}\text { Fructose } \\
\text { metabolism }\end{array}$ & 0.88 \\
\hline 4 & AT2G39730 & Rubisco activase & $\begin{array}{l}\text { Light activation of } \\
\text { rubisco }\end{array}$ & 1.21 \\
\hline 5 & AT5G15650 & UDP-arabinose mutase & $\begin{array}{l}\text { Arabinose } \\
\text { metabolism }\end{array}$ & 0.68 \\
\hline $6^{*}$ & AT1G65930 & $\begin{array}{l}\text { Cytosolic NADP+-dependent } \\
\text { isocitrate dehydrogenase }\end{array}$ & Redox & 0.22 \\
\hline $7 *$ & AT1G65930 & $\begin{array}{l}\text { Cytosolic NADP+-dependent } \\
\text { isocitrate dehydrogenase }\end{array}$ & Redox & 3.12 \\
\hline 8 & AT1G03475 & Coproporphyrinogen III oxidase & Redox & 1.15 \\
\hline 9 & AT5G09530 & Proline-rich protein 10 & Seed germination & 1.24 \\
\hline 10 & AT1G66200 & Cytosolic glutamate synthetase & $\begin{array}{l}\text { Glutamine } \\
\text { biosynthesis }\end{array}$ & 1.79 \\
\hline
\end{tabular}




\begin{tabular}{rrlll}
11 & ATCG00490 & Ribulose-bisphosphate carboxylase & $\begin{array}{l}\text { Carbon fixation of } \\
\text { photosynthesis }\end{array}$ & 0.79 \\
12 & AT3G44310 & Nitrilase 1 & $\begin{array}{l}\text { Nitrogen compound } \\
\text { metabolism }\end{array}$ & 1.37 \\
& & Redox & $\mathbf{1 . 2 6}$ \\
$\mathbf{1 3}$ & AT1G75280 & Isoflavone reductase & Redox & $\mathbf{1 . 1 6}$ \\
$\mathbf{1 4}$ & AT1G78380 & Glutathione s-transferase tau 19 & Response to UV & 0.67 \\
15 & AT1G20340 & DNA-damage resistance protein 112 & R \\
\hline
\end{tabular}

461 Proteins listed in bold black are differentially expressed and involved in redox. Points $6^{*}$ and $7^{*}$

462 indicate protein expression is significantly different from control. 'Expression' is calculated as the 463 ratio of the expression level in $\operatorname{brs} 1-1 D$ to the control WS2. 'Relative volume' values were 464 determined using ImageMaster Platinum Software. NO indicates the spot was missing in the 465 corresponding gel. For each sample, triplicate biological repeats of 2-DE were performed using 466 independent protein preparations.

\section{Additional files}

468 Additional file 1: Supplementary Table S1. Identification of DEGs in BRS1 mutants.

469 Supplementary Table S2. Proteins identified by BRS1-GFP pull-down.

470 Additional file 2: Supplementary Figure S1. The expression analysis of BRS1

471 homologs in wildtype and BRS1 mutants. RNA sequencing was used to calculate the 472 expression levels (FPKM value) of five BRS1 homologs: SCPL22, SCPL23, SCPL25, 473 SCPL26 and SCPL27 in WS2, brs 1-1 and brs1-1D plants. Mean \pm SD is shown. The 474 asterisks indicate a statistically significant difference (Student's t-test, $* \mathrm{P}<0.05$ ). 